This item was submitted to Loughborough's Research Repository by the author.

Items in Figshare are protected by copyright, with all rights reserved, unless otherwise indicated.

\title{
Predictive control for general nonlinear systems using approximation
}

PLEASE CITE THE PUBLISHED VERSION

PUBLISHER

(c) Institution of Electrical Engineers

LICENCE

CC BY-NC-ND 4.0

REPOSITORY RECORD

Chen, Wen-Hua. 2008. "Predictive Control for General Nonlinear Systems Using Approximation”. figshare. https://hdl.handle.net/2134/3798. 
This item was submitted to Loughborough's Institutional Repository (https://dspace.lboro.ac.uk/) by the author and is made available under the following Creative Commons Licence conditions.

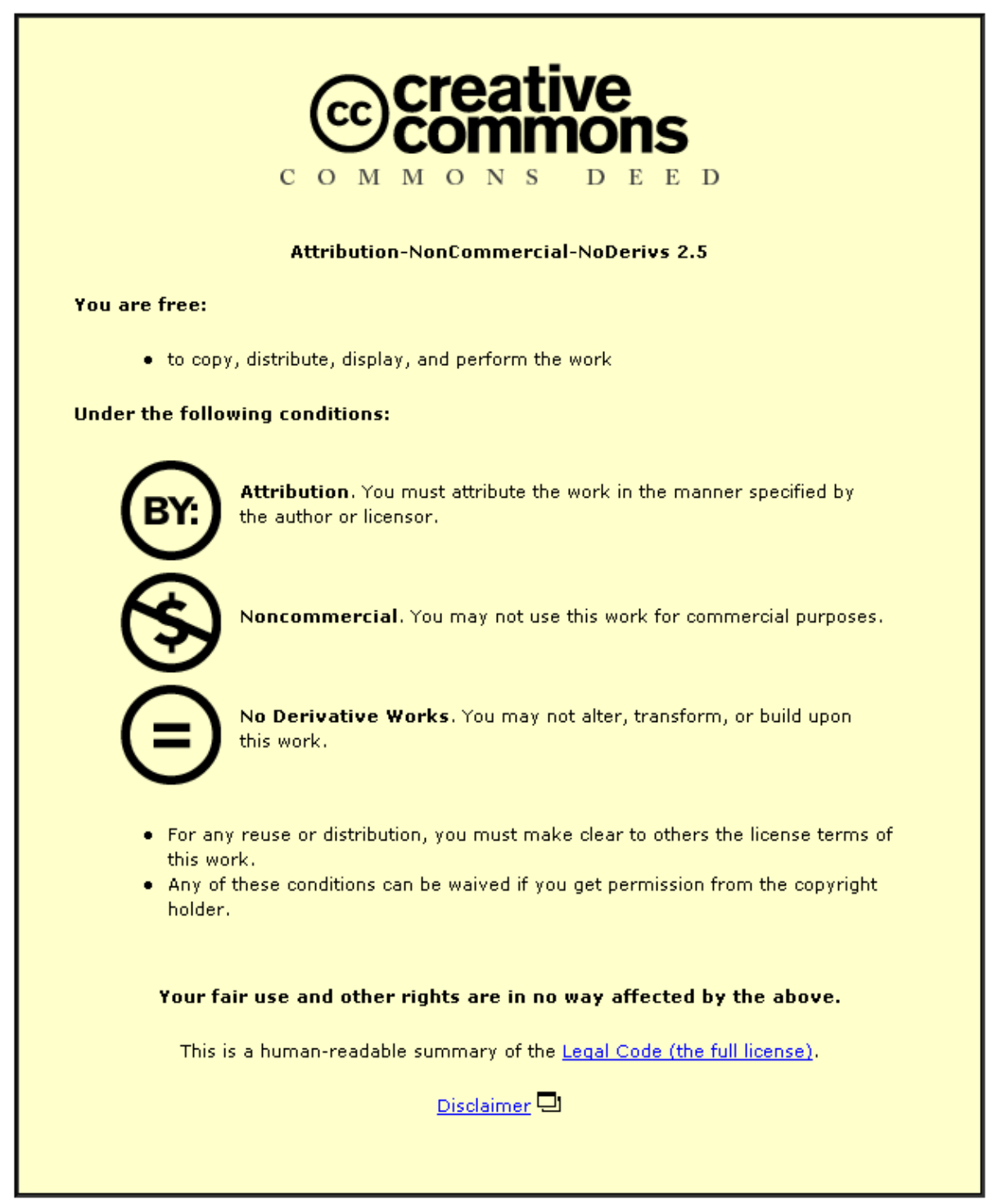

For the full text of this licence, please go to: http://creativecommons.org/licenses/by-nc-nd/2.5/ 


\title{
Predictive Control of General Nonlinear Systems Using Approximation
}

\author{
Wen-Hua Chen \\ Department of Aeronautical and Automotive Engineering \\ Loughborough University \\ Loughborough, Leicestershire LE11 3TU \\ U.K \\ Tel.: +44 (0) 1509227230 \\ Fax: +44 (0) 1509227275 \\ Email: W.Chen@lboro.ac.uk
}

October 1, 2003

\begin{abstract}
This paper addresses a tracking problem for general nonlinear systems using model predictive control (MPC). After approximating the tracking error in the receding horizon by its Taylor series expansion to any specified order, an analytic solution to the MPC is developed and a closed-form nonlinear predictive controller is presented. Different from other nonlinear model predictive control (NMPC), there is a built-in integral action in the developed scheme and the implementation issues are discussed. Further more, it is pointed out that the proposed NMPC derived using approximation can stablise the original nonlinear systems if certain condition, which can be met by properly choosing predictive times and the order for Taylor expansion, is satisfied. Simulation demonstrates the effectiveness of the proposed NMPC.
\end{abstract}




\section{Introduction}

Approximation of nonlinear systems by series expansion provides a powerful technique to tackle nonlinear systems. Series expansion linearisation theory is well established but is strictly confined to dynamic analysis, locally to a single trajectory or an equilibrium operating point, of smooth nonlinear systems. Frozen-input technique extends local linearisation theory from a single equilibrium point to a family of equilibrium points. This lays the foundation for several widespread control methods for nonlinear systems such as gain scheduling. However there are two main shortcomings in these techniques: One is that the linearisation is restricted to around equilibria, and the other is that the stability property is mainly considered and little insight into other dynamic behaviours is provided [1]. It was pointed out that while indicating stability, the linearisation provides, in general, somewhat poor indication of the time response of the original nonlinear system. Several approaches have been proposed to overcome these shortcomings such as local model networks [2], local control networks [3, 4] and velocity based linearisation [5]. In these approaches, the linearisation can be carried out not only on equilibria but also on any operating point, and the transient dynamics can be improved.

To develop model predictive control (MPC) for nonlinear systems, another approach to approximation of dynamics behaviours of a nonlinear system was adopted in $[6,7]$, where instead of linearisation of a nonlinear system around equilibria or offequilibria, the output of a nonlinear system is approximated by high series expansion. Although approximation of the output of a nonlinear system rather than the nonlinear system itself might render the stability analysis of the nonlinear system more difficult, it can provide direct insight into other dynamic properties like transient response. A nonlinear MPC (NMPC) algorithm was developed in $[6,7]$ using Taylor series expansion.

Similar to other NMPC algorithms (for example, see $[8,9,10]$ ), a differential optimisation problem has to been solved for the NMPC algorithm developed in $[6,7]$, which imposes two main obstacles in engineering implementation. One is the optimality of the online optimisation and the other is the sampling time restriction. In general, the on-line optimisation involved in NMPC is non-convex, which implies that

the optimisation procedure might be terminated at a local minimum. Consequently 
poor performance even instability might be resulted after the control sequence yielded by the on-line optimiser is implemented. The on-line nonlinear optimisation also imposes heavy computational burden, which requires extensive computing power and long sampling time in engineering implementation. For a system with fast dynamics, this problem becomes even more challenging due to the fast sampling requirement.

The best way to address these difficulties arising in predictive control of nonlinear systems is to pursue analytic approach, where it is intended to develop a closed form NMPC and online optimisation is not required. In the NMPC algorithm in [7], the optimality condition gives a set of highly nonlinear equations in terms of control and various derivatives of control (depending on the order for Taylor series expansion). It is very unlikely (if not impossible) to find the analytic optimal solution and actually it is also very difficult to find numerical optimal solution by using nonlinear optimization algorithms since it is, in general, non-convex. To obtain an analytic solution, the constraint on the order of Taylor series expansion is imposed, that is, the control order is limited to be zero, or the nonlinear system is only expanded by Taylor series expansion to its relative degree [6]. In this case, the control effort appears linearly in the optimality condition and there are no derivatives of the control effort due to zero control order. Then the analytic solution for nonlinear MPC was found, and it is also found that the nonlinear system is linearised by the derived analytic MPC law and the relationship between this analytic nonlinear control law and feedback linearisation was established in [11]. Similar idea was employed in $[12,13]$ independently to develop analytic NMPC using Taylor expansion and the relationships between the analytic control laws they developed and the feedback linearisation technique were also explored. In all these studies, the control order is restricted to be zero and there is no stability analysis for the developed algorithms.

The drawback of restricting the control order being zero is obvious. This implies the control effort to be optimized in the receding horizon is constant, which is a very restrictive requirement. In many cases, this implies that the nonlinear system can only be approximated by Taylor series expansion to its relative degree. With this limitation, it is understandable that poor performance might be resulted. Actually [14] shown that a nonlinear system with high relative degree under the NMPC law developed in this way is unstable, no matter how small the receding horizon is chosen. 
The first attempt to develop analytic solution of NMPC for arbitrary control order was made in [6]. Since a set of highly nonlinear equations derived from the optimality condition have to be solved to find analytic solution for MPC, two simplifications are used: one is the control weighting is not included in the performance index and the other is that control order is chosen as the difference between the order for Taylor expansion and the relative degree (the price for these two simplifications is that, unlike the original NMPC algorithm [7], the analytic MPC law is not applicable for nonlinear systems with unstable zero dynamics). Then an analytic NMC was derived on the assumption that the control variables appear in the optimality condition linearly, which, unfortunately, is not true for arbitrarily chosen Taylor expansion order. To find the analytic solution for NMPC, a set of very complicated nonlinear coupling equations in terms of control effort and its derivatives, which is derived from the optimal condition, still has to be solved. The analytic solution of the nonlinear coupling equations and then the closed-form NMPC for nonlinear systems approximated by Taylor series expansion to arbitrarily chosen order was found in [14]. Furthermore, the stability result for analytic NMPC was first established, and the influence of the control order and predictive horizon on the transient performance was investigated. This also implies that the closed form MPC developed based on the Taylor series expansion can approach to the optimal MPC for original nonlinear systems with any specified accuracy. This work was further extended to nonlinear systems with ill-defined relative degree [15]. All the existing analytic NMPC algorithms are confined to affine systems.

This paper further develops a closed-form NMPC for general nonlinear systems using the above output approximation technique. An analytic solution to NMPC for the tracking problem of general nonlinear systems is presented, where the tracking error in the receding horizon is approximated by its Taylor series expansion to any order. Stability of the proposed NMPC is established. Different from the NMPC schemes for affine systems, there is a built-in integral action in the control structure proposed in this paper and the implementation issues are discussed. 


\section{Predictive control of general nonlinear systems}

To simplify the notation and concentrate on the main contribution of this paper, only single-input-single-output (SISO) nonlinear systems are considered and most of the results developed in this paper are ready to extend to multivariable general nonlinear systems. An SISO general nonlinear system can be described by

$$
\left\{\begin{array}{l}
\dot{\boldsymbol{x}}(t)=\boldsymbol{f}(\boldsymbol{x}(t), u(t)) \\
y(t)=h(\boldsymbol{x}(t))
\end{array}\right.
$$

where $\boldsymbol{x} \in \mathbb{R}^{n}, u \in \mathbb{R}$ and $y \in \mathbb{R}$ are state, input and output respectively.

For the sake of simplicity, the following notation is introduced in this paper:

$$
\begin{gathered}
\mathcal{D}_{\boldsymbol{f}_{x}} h(\boldsymbol{x})=\frac{\partial h(\boldsymbol{x})}{\partial \boldsymbol{x}} \boldsymbol{f}(\boldsymbol{x}, u) \\
\mathcal{D}_{\boldsymbol{f}_{x}}^{k} h(\boldsymbol{x})=\frac{\partial \mathcal{D}_{\boldsymbol{f}_{x}}^{k-1} h(\boldsymbol{x})}{\partial \boldsymbol{x}} \boldsymbol{f}(\boldsymbol{x}, u), \text { for } k>1
\end{gathered}
$$

and

$$
\mathcal{D}_{u} \mathcal{D}_{\boldsymbol{f}_{x}}^{k} h(\boldsymbol{x})=\frac{\partial \mathcal{D}_{\boldsymbol{f}_{x}}^{k} h(\boldsymbol{x})}{\partial u},
$$

To develop our results, the following assumptions are imposed on the system (1):

A1: The general nonlinear system (1) is sufficiently differentiable with respect to time to any order;

A2: $\boldsymbol{f}(0,0)=0$;

A3: $\mathcal{D}_{u} \mathcal{D}_{\boldsymbol{f}_{x}}^{k} h(\boldsymbol{x})=0$, for $k=1, \ldots, \mu-1$, and $\mathcal{D}_{u} \mathcal{D}_{\boldsymbol{f}_{x}}^{\mu} h(\boldsymbol{x}) \neq 0$, for all $\boldsymbol{x}$ and $u$

Assumption A1 implies that the nonlinear system (1) can be approximated by its Taylor series expansion to any specified accuracy. Assumption A2 means that the origin is an equilibrium of the nonlinear system when there is no control. Assumption A3 is similar to the well defined relative degree for affine nonlinear systems, although, as will be shown later, the derivative of the control is used in controller design for general nonlinear systems.

Different from most of the conventional NMPC algorithms for nonlinear systems $[8,9,10]$, the tracking problem is considered in this paper, following [7, 14]. That is, a predictive controller is designed such that the output, $y$, optimally follows an 
reference signal $\omega$ in terms of a given performance index. The most widely used quadratic performance index is adopted in this paper, given by

$$
J=\frac{1}{2} \int_{T_{1}}^{T_{2}} e(t+\tau)^{2} d \tau
$$

where $T_{1}$ and $T_{2}$ are the lower and upper predictive times respectively. $e$ is the tracking error, defined as

$$
e(t+\tau)=y(t+\tau)-\omega(t+\tau)
$$

\section{Tracking error approximation}

Suppose that the output of the nonlinear system in the prediction horizon is approximated by its Taylor series expansion up to order $r \geq \mu$. One has

$$
y(t+\tau) \approx y(t)+\tau \dot{y}(t)+\ldots+\frac{\tau^{r}}{r !} y^{[r]}(t), \quad T_{1} \leq \tau \leq T_{2}
$$

The derivatives of the output required in the approximation are given by

$$
\begin{aligned}
\dot{y}(t) & =\frac{\partial h(\boldsymbol{x})}{\partial \boldsymbol{x}} \dot{\boldsymbol{x}} \\
& =\frac{\partial h(\boldsymbol{x})}{\partial \boldsymbol{x}} \boldsymbol{f}(\boldsymbol{x}, u) \\
& =\mathcal{D}_{\boldsymbol{f}_{x}} h(\boldsymbol{x})
\end{aligned}
$$

and

$$
\begin{aligned}
\ddot{y}(t) & =\frac{\partial}{\partial \boldsymbol{x}}\left(\frac{\partial h(\boldsymbol{x})}{\partial \boldsymbol{x}} \boldsymbol{f}(\boldsymbol{x}, u)\right) \dot{\boldsymbol{x}}+\frac{\partial}{\partial u}\left(\frac{\partial h(\boldsymbol{x})}{\partial \boldsymbol{x}} \boldsymbol{f}(\boldsymbol{x}, u)\right) \dot{u} \\
& =\mathcal{D}_{\boldsymbol{f}_{x}}^{2} h(\boldsymbol{x})
\end{aligned}
$$

The last equality follows form the notation in (2), (3) and Assumption A3. Similarly, one has

$$
y^{[k]}(t)=\mathcal{D}_{\boldsymbol{f}_{x}}^{k} h(\boldsymbol{x}), \text { for } k=3, \ldots, \mu
$$

and

$$
\begin{aligned}
y^{[\mu+1]}(t) & =\frac{\partial \mathcal{D}_{\boldsymbol{f}_{x}}^{\mu}}{\partial \boldsymbol{x}} \boldsymbol{f}(\boldsymbol{x}, u)+\frac{\partial \mathcal{D}_{\boldsymbol{f}_{x}}^{\mu}}{\partial u} \dot{u} \\
& =\mathcal{D}_{\boldsymbol{f}_{x}}^{\mu+1} h(\boldsymbol{x})+\mathcal{D}_{u} \mathcal{D}_{\boldsymbol{f}_{x}}^{\mu} h(\boldsymbol{x}) \dot{u}
\end{aligned}
$$


Differentiation of $y^{[\mu+1]}$ with respect to time $t$ and substitution of the system's dynamics gives

$$
\begin{aligned}
y^{[\mu+2]}(t)= & \frac{\partial \mathcal{D}_{\boldsymbol{f}_{x}}^{\mu+1} h(\boldsymbol{x})}{\partial \boldsymbol{x}} \boldsymbol{f}(\boldsymbol{x}, u)+\frac{\partial \mathcal{D}_{\boldsymbol{f}_{x}}^{\mu+1} h(\boldsymbol{x})}{\partial u} \dot{u}+\frac{\partial \mathcal{D}_{u} \mathcal{D}_{\boldsymbol{f}_{x}}^{\mu} h(\boldsymbol{x})}{\partial \boldsymbol{x}} \boldsymbol{f}(\boldsymbol{x}, u) \dot{u}+ \\
& \frac{\partial \mathcal{D}_{u} \mathcal{D}_{\boldsymbol{f}_{x}}^{\mu} h(\boldsymbol{x})}{\partial u} \dot{u}^{2}+\mathcal{D}_{u} \mathcal{D}_{\boldsymbol{f}_{x}}^{\mu} h(\boldsymbol{x}) \ddot{u} \\
= & \mathcal{D}_{\boldsymbol{f}_{x}}^{\mu+2} h(\boldsymbol{x})+\mathcal{D}_{u} \mathcal{D}_{\boldsymbol{f}_{x}}^{\mu} h(\boldsymbol{x}) \ddot{u}+z_{1}(\boldsymbol{x}, u, \dot{u})
\end{aligned}
$$

where

$$
z_{1}(\boldsymbol{x}, u, \dot{u})=\frac{\partial \mathcal{D}_{\boldsymbol{f}_{x}}^{\mu+1} h(\boldsymbol{x})}{\partial u} \dot{u}+\frac{\partial \mathcal{D}_{u} \mathcal{D}_{\boldsymbol{f}_{x}}^{\mu} h(\boldsymbol{x})}{\partial \boldsymbol{x}} \boldsymbol{f}(\boldsymbol{x}, u) \dot{u}+\frac{\partial \mathcal{D}_{u} \mathcal{D}_{\boldsymbol{f}_{x}}^{\mu} h(\boldsymbol{x})}{\partial u} \dot{u}^{2}
$$

Repeating the above procedure, the higher order derivatives can be calculated until the $r$ th order derivative, which is given by

$$
y^{[r]}(t)=\mathcal{D}_{\boldsymbol{f}_{x}}^{r} h(\boldsymbol{x})+\mathcal{D}_{u} \mathcal{D}_{\boldsymbol{f}_{x}}^{\mu} h(\boldsymbol{x}) u^{[r-\mu]}+z_{r-\mu-1}\left(\boldsymbol{x}, u, \dot{u}, \ldots, u^{[r-\mu-1]}\right)
$$

where $z_{r-\mu-1}\left(\boldsymbol{x}, u, \dot{u}, \ldots, u^{[r-\mu-1]}\right)$ is a complicated nonlinear fucntion of $\boldsymbol{x}, u, \dot{u}, \ldots, u^{[r-\mu-1]}$.

Invoking (8-14) into (7), the output in the receding horizon is approximated by its Taylor expansion to the order $r$ as

$$
y(t+\tau) \approx \tau \boldsymbol{Y}, \quad T_{1} \leq \tau \leq T_{2}
$$

where

$$
\boldsymbol{\tau}=\left[\begin{array}{llll}
1 & \tau & \ldots & \frac{\tau^{r}}{r !}
\end{array}\right]
$$

and

$$
\begin{aligned}
& \boldsymbol{Y}(t)=\left[\begin{array}{llllllll}
y^{[0]}(t) & y^{[1]}(t) & \cdots & y^{[\mu]}(t) & y^{[\mu+1]}(t) & y^{[\mu+2]}(t) & \cdots & y^{[r]}(t)
\end{array}\right]^{T} \\
& =\left[\begin{array}{c}
h(\boldsymbol{x}) \\
\mathcal{D}_{\boldsymbol{f}_{x}} h(\boldsymbol{x}) \\
\vdots \\
\mathcal{D}_{\boldsymbol{f}_{x}}^{\mu} h(\boldsymbol{x}) \\
\mathcal{D}_{\boldsymbol{f}_{x}}^{\mu+1} h(\boldsymbol{x}) \\
\mathcal{D}_{\boldsymbol{f}_{x}}^{\mu+2} h(\boldsymbol{x}) \\
\vdots \\
\mathcal{D}_{\boldsymbol{f}_{x}}^{r} h(\boldsymbol{x})
\end{array}\right]+\left[\begin{array}{c}
0 \\
0 \\
\vdots \\
0 \\
\mathcal{D}_{u} \mathcal{D}_{\boldsymbol{f}_{x}}^{\mu} h(\boldsymbol{x}) \dot{u} \\
\mathcal{D}_{u} \mathcal{D}_{\boldsymbol{f}_{x}}^{\mu} h(\boldsymbol{x}) \ddot{u}+z_{1}(\boldsymbol{x}, u, \dot{u}) \\
\vdots \\
\mathcal{D}_{\boldsymbol{f}_{x}}^{\mu} h(\boldsymbol{x}) u^{[r-\mu]}+z_{r-\mu-1}\left(\boldsymbol{x}, u, \dot{u}, \ldots, u^{[r-\mu-1]}\right)
\end{array}\right]
\end{aligned}
$$


In the same fashion, the command $\omega(t+\tau)$ in the receding horizon can also be approximated by its Taylor series expansion to order $r$ as

$$
\omega(t+\tau)=\tau \omega
$$

where

$$
\boldsymbol{\omega}(t)=\left[\begin{array}{lllllll}
\omega^{[0]}(t) & \omega^{[1]}(t) & \cdots & \omega^{[\mu]}(t) & \omega^{[\mu+1]}(t) & \cdots & \omega^{[r]}(t)
\end{array}\right]^{T}
$$

\section{Nonlinear predictive controller}

After approximating the output of the nonlinear system (1) and the reference to be tracked by series expansion, the tracking error then can be calculated by

$$
\begin{aligned}
e(t+\tau) & =y(t+\tau)-\omega(t+\tau) \\
& \approx \boldsymbol{\tau}(\boldsymbol{Y}(t)-\boldsymbol{\omega}(t))
\end{aligned}
$$

Thus the predictive control performance (5) can be approximated by

$$
\begin{aligned}
J & \approx \frac{1}{2} \int_{T_{1}}^{T_{2}}(\boldsymbol{Y}(t)-\boldsymbol{\omega}(t))^{T} \boldsymbol{\tau}^{T} \boldsymbol{\tau}(\boldsymbol{Y}(t)-\boldsymbol{\omega}(t)) d \tau \\
& =\frac{1}{2}(\boldsymbol{Y}(t)-\boldsymbol{\omega}(t))^{T} \int_{T_{1}}^{T_{2}} \boldsymbol{\tau}^{T} \boldsymbol{\tau} d \tau(\boldsymbol{Y}(t)-\boldsymbol{\omega}(t)) \\
& =\frac{1}{2}(\boldsymbol{Y}(t)-\boldsymbol{\omega}(t))^{T} \boldsymbol{T}(\boldsymbol{Y}(t)-\boldsymbol{\omega}(t))
\end{aligned}
$$

where

$$
\begin{aligned}
\mathcal{T} & =\int_{T_{1}}^{T_{2}} \boldsymbol{\tau}^{T} \boldsymbol{\tau} d \tau \\
& =\left[\begin{array}{cccc}
T_{2}-T_{1} & \frac{T_{2}^{2}-T_{1}^{2}}{2} & \ldots & \frac{T_{2}^{r+1}-T_{1}^{r+1}}{r+1) !} \\
\frac{T_{2}^{2}-T_{1}^{2}}{2} & \frac{T_{2}^{3}-T_{1}^{3}}{3} & \ldots & \frac{T_{2}^{r+2}-T_{1}^{r+2}}{r !(r+2)} \\
\ldots & \ldots & \ldots & \ldots \\
\frac{T_{2}^{r+1}-T_{1}^{r+1}}{(r+1) !} & \frac{T_{2}^{r+2}-T_{1}^{r+2}}{r !(r+2)} & \ldots & \frac{T_{2}^{2 r+1}-T_{1}^{2 r+1}}{r ! r !(2 r+1)}
\end{array}\right]
\end{aligned}
$$

At the time $t, \mathrm{MPC}$ attempts to find the optimal control profile in the receding horizon, $u(t+\tau), 0 \leq \tau \leq T_{2}$, to minimise the tracking error. After the nonlinear system (1) is approximated by its Taylor series expansion up to the order $r$, the 
highest derivative of the control required is $r-\mu$. Hence all the control profile in the receding horizon can be parametrarized by

$$
u(t+\tau)=u(t)+\tau \dot{u}(t)+\cdots+\frac{\tau^{r-\mu}}{(r-\mu) !} u^{[r-\mu]}(t)
$$

Therefore, instead of minimising the performance index $J$ in terms of the control profile $u(t+\tau), 0 \leq \tau \leq T_{2}$ directly, the performance index $J$ can be minimised in terms of variables $u(t), \dot{u}(t), \ldots, u^{[r-\mu]}(t)$. However, for a general nonlinear under consideration as in (1), the control $u$ does not appear in a linear manner and it is difficult to give the explicit solution for $u$. To avoid this problem, the control $u$ is considered as a new state variable, and the performance $J$ is minimised in terms of $\dot{u}(t), \ldots, u^{[r-\mu]}(t)$. In the later of this paper, we will discuss the implementation issue for the proposed controller.

The necessary condition for the optimality is given by

$$
\frac{\partial J}{\partial \boldsymbol{u}}=0
$$

where

$$
\boldsymbol{u}=\left[\dot{u}(t), \ldots, u^{[r-\mu]}(t)\right]^{T}
$$

Theorem 1: Consider a general nonlinear system (1) satisfying Assumption A1-A3 and with the tracking performance index (5). When the tracking error is approximated by its Taylor series expansion up to order $r \geq \mu$, the NMPC is given by

$$
u(t)=\int \dot{u}(\sigma) d \sigma
$$

where

$$
\begin{gathered}
\dot{u}(\sigma)=-\left(\mathcal{D}_{u} \mathcal{D}_{\boldsymbol{f}_{x}}^{\mu} h(\boldsymbol{x})\right)^{-1}\left(\boldsymbol{K} \boldsymbol{M}_{\mu}(\sigma)+\mathcal{D}_{\boldsymbol{f}_{x}}^{\mu+1} h(\boldsymbol{x})-\omega^{[\mu+1]}(\sigma)\right) \\
\boldsymbol{M}_{\mu}(\sigma)=\left(\begin{array}{c}
h(\boldsymbol{x})-\omega(\sigma) \\
\mathcal{D}_{\boldsymbol{f}_{x}}^{1} h(\boldsymbol{x})-\omega^{[1]}(\sigma) \\
\ldots \\
\mathcal{D}_{\boldsymbol{f}_{x}}^{\mu} h(\boldsymbol{x})-\omega^{[\mu]}(\sigma)
\end{array}\right)
\end{gathered}
$$

and $\boldsymbol{K} \in \mathbb{R}^{\mu+1}$ is the first row of the matrix $\mathcal{T}_{r r}^{-1} \mathcal{T}_{\mu r}^{T}$,

$$
\mathcal{T}_{\mu r}=\left[\begin{array}{ccc}
\frac{T_{2}^{\mu+2}-T_{1}^{\mu+2}}{(\mu+1) !(\mu+2)} & \cdots & \frac{T_{2}^{2 \mu+2}-T_{1}^{2 \mu+2}}{\mu !(\mu+1) !(2 \mu+2)} \\
\vdots & \vdots & \vdots \\
\frac{T_{2}^{r+1}-T_{1}^{r+1}}{(r+1) !} & \cdots & \frac{T_{2}^{r+\mu+1}-T_{1}^{r+\mu+1}}{\mu ! r !(r+\mu+1)}
\end{array}\right],
$$




$$
\mathcal{T}_{r r}=\left[\begin{array}{ccc}
\frac{T_{2}^{2 \mu+3}-T_{1}^{2 \mu+3}}{(\mu+1) !(\mu+1) !(2 \mu+3)} & \cdots & \frac{T_{2}^{r+\mu+2}-T_{1}^{r+\mu+2}}{(\mu+1) ! r !(r+\mu+2)} \\
\vdots & \vdots & \vdots \\
\frac{T_{2}^{r+\mu+2}-T_{1}^{r+\mu+2}}{(\mu+1) ! r !(r+\mu+2)} & \cdots & \frac{T_{2}^{2 r+1}-T_{1}^{2 r+1}}{r ! r !(2 r+1)}
\end{array}\right],
$$

Proof: See Appendix.

Remark 1: Theorem 1 presents an analytic solution to the NMPC problem for general nonlinear systems using approximation and the NMPC is given in a closedform. The existing results for analytic NMPC is extended from affine nonlinear systems to general nonlinear systems. This controller overcomes the difficulties imposed by the on-line nonlinear optimisation involved in NMPC such as attaining local minimum and long sampling time requirement. The NMPC developed in this paper is suboptimal for the original NMPC problem. Since there is no restriction on the order for Taylor series expansion, theoretically the analytic solution developed by approximation can approach to the solution to the original NMPC in any specified accuracy.

\subsection{Structure of predictive control}

Since the closed-form NMPC for the general nonlinear system is derived, there is no on-line optimisation required. Actually from the control law (26) and (27), one can see that it is a nonlinear state variable feedback control law. After the order for Taylor series expansion, the order $\mu$ and the predictive times $T_{1}, T_{2}$ are chosen, the control gain $\boldsymbol{K}$ can be calculated off-line.

The block diagram of the proposed NMPC scheme is shown in Figure 1. Different from previous analytic nonlinear predictive control schemes for affine systems, the derivative of the control action is calculated using the control law (27) based on the state variable, the current control and the reference, and then the control action is obtained by integrating it with respect to time.

\subsection{The choice of the initial control}

In the implementation of the above control strategy for general nonlinear systems, the initial control $u(0)$ should be specified. Suppose that at the initial state, the system is at its equilibrium. It follows form Assumption A3 that the initial control $u(0)$ should be chosen as zero. 


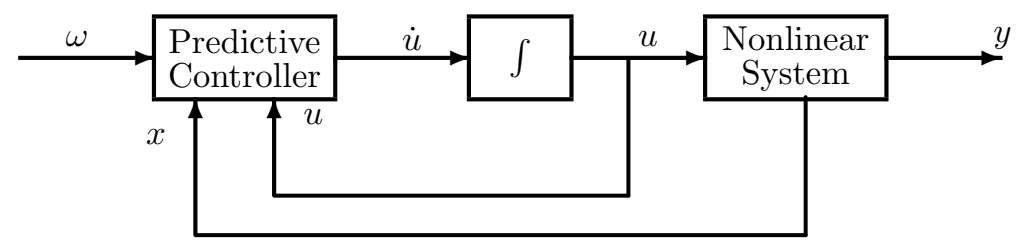

Figure 1: Structure of predictive control for general nonlinear systems

\section{$5 \quad$ Stability Analysis}

Substituting the control law (27) into (11) gives

$$
y^{[\mu+1]}(t)=\mathcal{D}_{\boldsymbol{f}_{x}}^{\mu+1} h(\boldsymbol{x})-\left(\boldsymbol{K} \boldsymbol{M}_{\mu}(t)+\mathcal{D}_{\boldsymbol{f}_{x}}^{\mu+1} h(\boldsymbol{x})-\omega^{[\mu+1]}(t)\right)
$$

which implies that

$$
y^{[\mu+1]}(t)-\omega^{[\mu+1]}(t)+\boldsymbol{K} \boldsymbol{M}_{\mu}(t)=0
$$

Eq.(28) is used for calculating the control law which explicitly shows that $\dot{u}(t)$ depends on the state and the current control $u(t)$. Using the relationship in (8-10), Eq. (28) can also be written as

$$
\boldsymbol{M}_{\mu}=\left(\begin{array}{c}
y(t)-\omega(t) \\
\dot{y}(t)-\omega^{[1]}(t) \\
\ldots \\
y^{[\mu]}-\omega^{[\mu]}(t)
\end{array}\right)=\left(\begin{array}{c}
e(t) \\
\dot{e}(t) \\
\ldots \\
e^{[\mu]}(t)
\end{array}\right)
$$

Invoking (33) into the error dynamics (32) obtains

$$
e^{[\mu+1]}(t)+k_{\mu+1} e^{[\mu]}(t)+\cdots+k_{2} \dot{e}(t)+k_{1} e(t)=0
$$

where

$$
\boldsymbol{K}=\left[\begin{array}{ll}
k_{1} & \cdots k_{\mu+1}
\end{array}\right]
$$

$\mathcal{T}_{r \mu}$ and $\mathcal{T}_{r r}$ in (29) and (30) can be written as

$$
\begin{aligned}
\mathcal{T}_{\mu r}= & \operatorname{diag}\left\{\frac{T_{2}^{\mu+2}}{(\mu+1) !}, \frac{T_{2}^{\mu+3}}{(\mu+2) !}, \ldots, \frac{T_{2}^{r+1}}{r !}\right\}\left[\begin{array}{ccc}
\frac{1-{\frac{T_{1}}{T_{2}}}^{\mu+2}}{\mu+2} & \cdots & \frac{1-{\frac{T_{1}}{T_{2}}}^{2 \mu+2}}{2 \mu+2} \\
\vdots & \vdots & \vdots \\
\frac{1-{\frac{T}{T_{2}}}^{r+1}}{r+1} & \cdots & \frac{1-{\frac{T_{1}}{T_{2}}}_{r+\mu+1}^{r+\mu+1}}{}
\end{array}\right] \\
& \operatorname{diag}\left\{1, T_{2}, \ldots, \frac{T_{2}^{\mu}}{\mu !}\right\}
\end{aligned}
$$


and

$$
\begin{aligned}
& \mathcal{T}_{r r}=\operatorname{diag}\left\{\frac{T_{2}^{\mu+2}}{(\mu+1) !}, \frac{T_{2}^{\mu+3}}{(\mu+2) !}, \ldots, \frac{T_{2}^{r+1}}{r !}\right\}\left[\begin{array}{ccc}
\frac{1-{\frac{T_{1}}{T_{2}}}^{2 \mu+3}}{2 \mu+3} & \cdots & \frac{1-{\frac{T_{1}}{T_{2}}}^{r+\mu+2}}{r+\mu+2} \\
\vdots & \vdots & \vdots \\
\frac{1-{\frac{T_{1}}{T_{2}}}^{r+\mu+2}}{r+\mu+2} & \cdots & \frac{1-{\frac{T}{T_{2}}}^{2 r+1}}{2 r+1}
\end{array}\right] \\
& \operatorname{diag}\left\{\frac{T_{2}^{\mu+1}}{(\mu+1) !}, \frac{T_{2}^{\mu+2}}{(\mu+2) !}, \ldots, \frac{T_{2}^{r}}{r !}\right\}
\end{aligned}
$$

Let

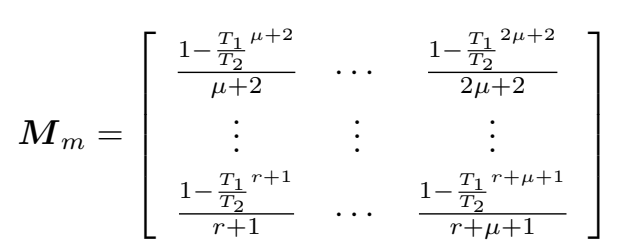

and

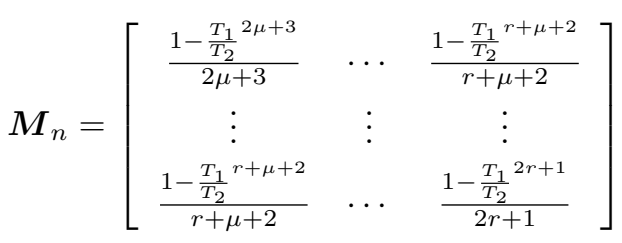

Then one has

$$
\begin{aligned}
\mathcal{T}_{r r}^{-1} \mathcal{T}_{r \mu}= & \operatorname{diag}\left\{T_{2}^{-(\mu+1)}(\mu+1) !, T_{2}^{-(\mu+2)}(\mu+2) !, \ldots, T_{2}^{-r} r !\right\} \boldsymbol{M}_{n}^{-1} \boldsymbol{M}_{m} \\
& \operatorname{diag}\left\{1, T_{2}, \ldots, \frac{T_{2}^{\mu}}{\mu !}\right\}
\end{aligned}
$$

Let $\boldsymbol{m}=\left[m_{1}, m_{2}, \ldots, m_{\mu+1}\right]$ denote the first row of the matrix $\boldsymbol{M}_{n}^{-1} \boldsymbol{M}_{m}$. Since the gain $\boldsymbol{K}$ is determined by the first row of the matrix $\boldsymbol{\mathcal { T }}_{r r}^{-1} \boldsymbol{\mathcal { T }}_{r \mu}$, it follows from (40) and (35) that

$$
k_{i}=m_{i} T_{2}^{-\mu-2+i} \frac{(\mu+1) !}{(i-1) !}, \quad i=1, \ldots, \mu+1
$$

where $0 !=1$. Substituting the gains into the error dynamics (34) yields

$$
\begin{aligned}
& e^{[\mu+1]}(t)+m_{\mu+1} T_{2}^{-1}(\mu+1) e^{[\mu]}(t)+m_{\mu} T_{2}^{-2}(\mu+1) \mu e^{[\mu]}(t)+\cdots+ \\
& +m_{2} T_{2}^{-\mu} \frac{(\mu+1) !}{1 !} \dot{e}(t)+m_{1} T_{2}^{-\mu-1}(\mu+1) ! e(t)=0
\end{aligned}
$$

One can conclude that the stability of the error dynamics is determined by the polynomial

$$
\begin{aligned}
& s^{\mu+1}+m_{\mu+1} T_{2}^{-1}(\mu+1) s^{\mu}+m_{\mu} T_{2}^{-2}(\mu+1) \mu s^{\mu-1}+\cdots+ \\
& +m_{\mu} T_{2}^{-\mu}(\mu+1) ! s+m_{1} T_{2}^{-\mu-1}(\mu+1) !=0
\end{aligned}
$$


i.e.,

$$
\begin{aligned}
& T_{2}^{\mu+1} s^{\mu+1}+m_{\mu+1}(\mu+1) T_{2}^{\mu} s^{\mu}+m_{\mu}(\mu+1) \mu T_{2}^{\mu-1} s^{\mu-1}+\cdots+ \\
& +m_{2}(\mu+1) ! T_{2} s+m_{1}(\mu+1) !=0
\end{aligned}
$$

Now using the transform $\widetilde{s}=T_{2} s$, the above polynomial becomes

$$
\begin{aligned}
& \widetilde{s}^{\mu+1}+m_{\mu+1}(\mu+1) \widetilde{s}^{\mu}+m_{\mu}(\mu+1) \mu \widetilde{s}^{\mu-1}+\cdots+ \\
& +m_{2}(\mu+1) ! \widetilde{s}+m_{1}(\mu+1) !=0
\end{aligned}
$$

The error dynamics (34) is stable if and only if all roots of the polynomial in (45) have negative real parts since the transform $\widetilde{s}=T_{2} s$ does not change the sign of the roots. Therefore, the stability of the error dynamics only depends on the coefficients $m_{i}, i=1, \ldots, \mu+1$, i.e., the first row of the matrix $\boldsymbol{M}_{n}^{-1} \boldsymbol{M}_{m}$.

However, to ensure the stability of the overall closed-loop system, the stability of the error dynamics alone is not enough. All the driven zero dynamics under the reference signal $\omega$ should be stable. One can conclude that under the assumption that the internal zero dynamics of the system (1) driven by $\omega$ are defined for all $t \geq 0$, bounded and uniformly asymptotically stable, the general nonlinear system (1) is stable under the NMPC (27) if all the roots of the polynomial (45) have negative real parts [16].

The foregoing stability analysis is summarised in Theorem 2 :

Theorem 2: Consider a general nonlinear system (1) satisfying Assumptions A1A3 and suppose that its internal zero dynamics driven by the reference $\omega$ are defined for all $t \geq 0$, bounded and uniformly asymptotically stable. The nonlinear system (1) under the NMPC (26), (27) is asymptotically stable if all the roots of the polynomial (45) have negative real parts.

Test of the stability of the error dynamics can be performed as follows: After the predictive times $T_{1}$ and $T_{2}$ are chosen and the order for Taylor expansion of the general nonlinear system is determined, calculate the matrix $\boldsymbol{M}_{n}$ and $\boldsymbol{M}_{m}$ according to (38) and (39). Then the first row of the matrix $\boldsymbol{M}_{n}^{-1} \boldsymbol{M}_{m}$ can be determined and, after substituting these coefficients to the polynomial (45), the stability can be tested.

The stability of the closed-loop system under the developed nonlinear control depends on the predictive times $T_{1}$ and $T_{2}$, the order $\mu$ that relates to the physical 
characteristics of the nonlinear system, and the order of Taylor series expansion. Further more, when $T_{1}$ is chosen as zero, it follows from (38) and (39) that the coefficients in the polynomial (45) do not depend on the predictive time $T_{2}$. This implies that in this case, the stability only depends on $\mu$ and the Taylor expansion order $r$. In general, the stability can be achieved by using a large $r$, i.e., approximating a general nonlinear system with Taylor series expansion to a high order [14].

Remark 2: The relationship between the analytic NMPC with the feedback linearisation for affine systems has been discussed in $[12,13,7]$. This paper shows that the general system (1) is also linearised by the developed analytic NMPC. This provides a new method to linearise general nonlinear systems using feedback, while minimising certain quadratic tracking performance specification.

\section{Illustrative example}

The NMPC developed in this paper is illustrated by a second order general nonlinear system, which was taken from $[6,7]$

$$
\left\{\begin{array}{l}
\dot{x}_{1}(t)=x_{2}(t)+\tanh (u(t)) \\
\dot{x}_{2}(t)=-x_{1}(t)+x_{2}^{2}(t)+u(t)
\end{array}\right.
$$

with the output

$$
y(t)=x_{1}(t)
$$

In order to design the NMPC developed in this paper, first the order $\mu$ needs to be calculated.

$$
\begin{aligned}
\mathcal{D}_{\boldsymbol{f}_{x}} h(\boldsymbol{x})= & \frac{\partial h(\boldsymbol{x})}{\partial \boldsymbol{x}} \boldsymbol{f}(\boldsymbol{x}, u) \\
= & {\left[\begin{array}{ll}
1 & 0
\end{array}\right]\left[\begin{array}{c}
x_{2}(t)+\tanh (u(t)) \\
-x_{1}(t)+x_{2}^{2}(t)+u(t)
\end{array}\right] } \\
= & x_{2}(t)+\tanh (u(t)) \\
& \mathcal{D}_{u} \mathcal{D}_{\boldsymbol{f}_{x}} h(\boldsymbol{x})=\frac{1}{\cosh ^{2}(u)}
\end{aligned}
$$

which is not equal to zero for all $x$ and $u$. Hence $\mu=1$.

It follows from Theorem 1 that the NMPC law is given by

$$
\dot{u}(t)=\frac{1}{\cosh ^{2}(u)}\left(-\left(-x_{1}(t)+x_{2}^{2}(t)+u\right)+\omega^{[2]}(t)+k_{2}(\dot{\omega}(t)-\dot{y}(t))+k_{1}(\omega(t)-y(t))\right)
$$




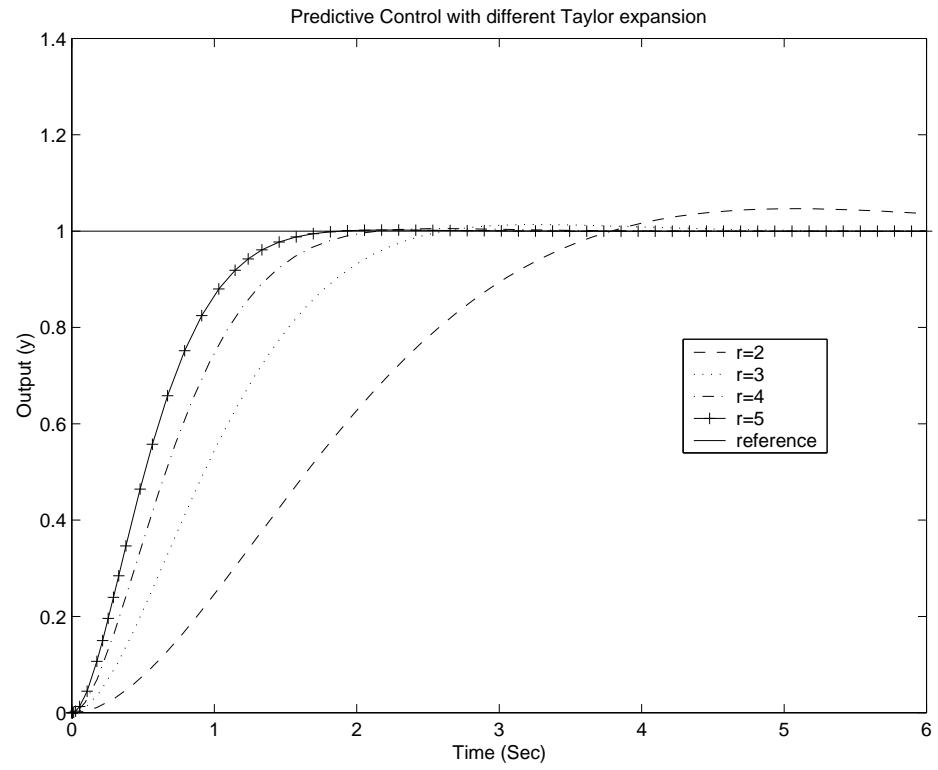

Figure 2: The tracking performance under the nonlinear NMPC with series expansion to different orders

where $k_{1}$ and $k_{2}$ are control gains determined by (41).

To investigate the performance of the NMPC developed using approximation, the first test is to fix the predictive times but approximate the nonlinear system with Taylor series expansion to different order. Control performance with the Taylor expansion order $\mathrm{r}=2,3,4,5$ are shown in Figure 2 and 3, where the predictive times are chosen as $T_{1}=1(\mathrm{~s})$ and $T_{2}=2(\mathrm{~s})$.

The second test is to investigate the influence of the choice of the predictive times. The order for Taylor series expansion is chosen as $r=5$ for all the simulation and various predictive times are considered. The tracking performance and the control profile are shown in Figure 4 and 5. In the simulation study, the length of the predictive horizon is kept as the same, i.e., $T_{2}-T_{1}=1(\mathrm{~s})$. It can be seen from Figure 4 and 5 that with the same order for Taylor series expansion, the rising time increases with the increase of the predictive times, while the control effort decreases with the increase of the predictive times. 


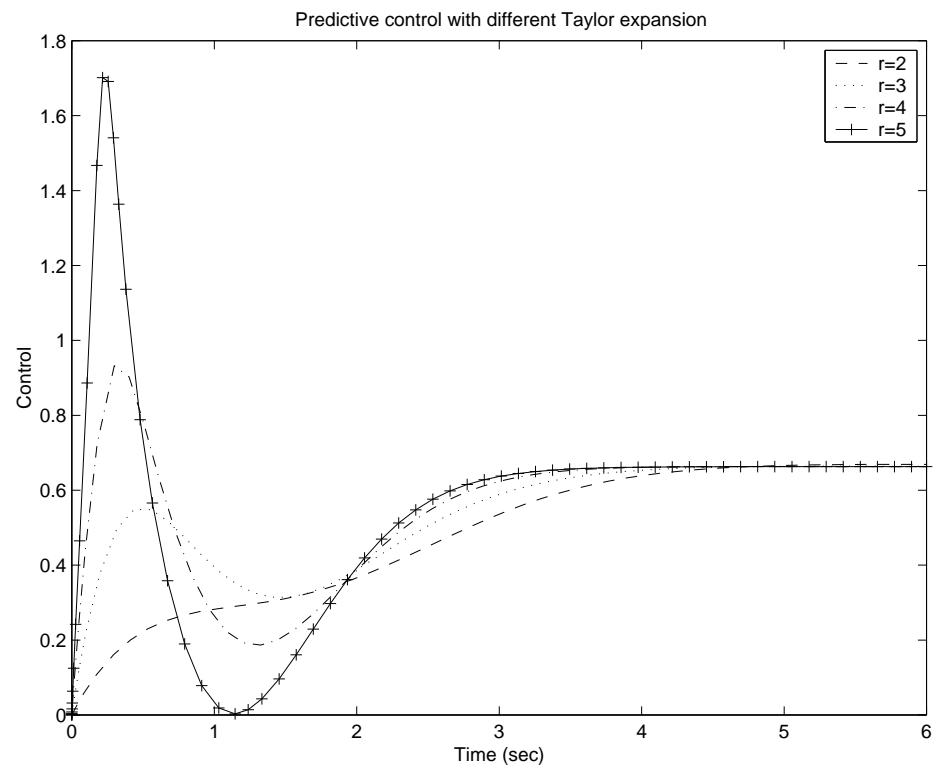

Figure 3: The time histories of control for series expansion to different orders

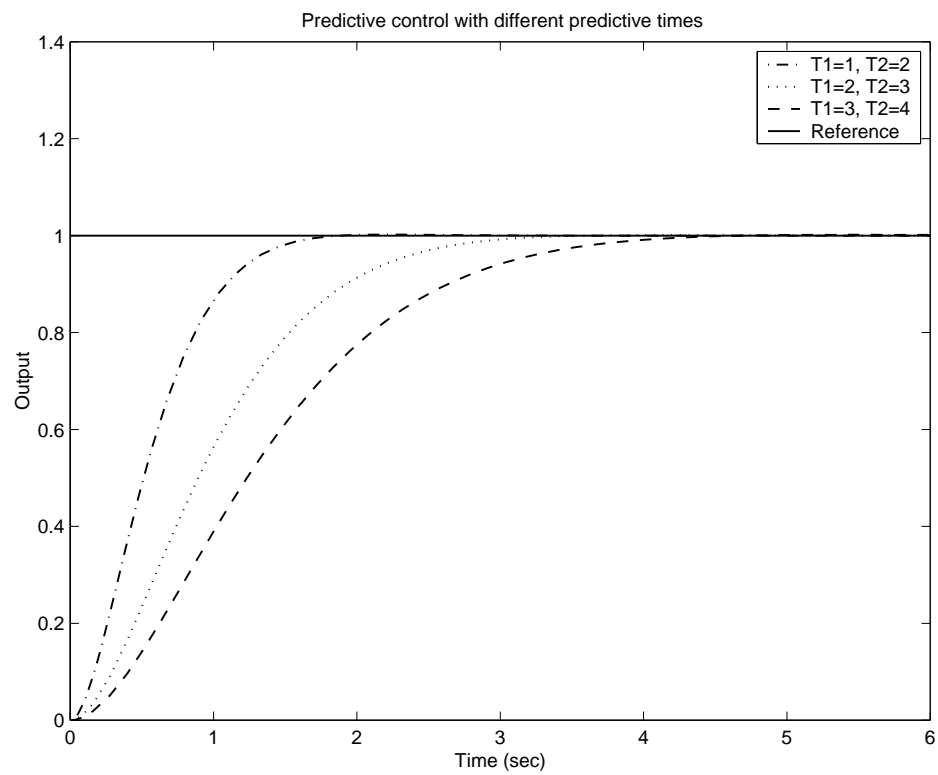

Figure 4: The tracking performance with 5th order Taylor series expansion and different predictive times 


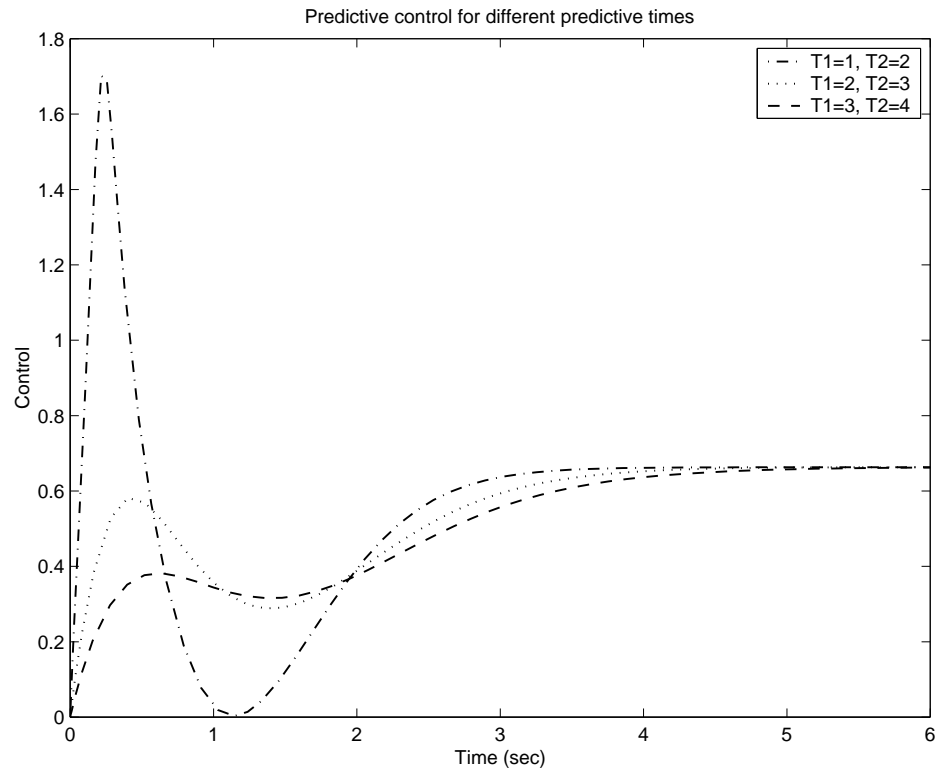

Figure 5: Control profiles for 5th order Taylor series expansion and different predictive times

\section{Conclusion}

For a general nonlinear system, an analytic solution to NMPC was developed in this paper by tracking error approximation. In this setting, the tracking performance is defined by a receding horizon performance index in terms of the tracking error and then the tracking error is approximated by its Taylor series expansion. Since there is no restriction on the order for series expansion, theoretically the analytic solution presented in this paper can approach to the original nonlinear predictive control problem in any specified accuracy. The implementation issues including the controller structure and the choice of the initial control action are discussed. It is shown that the NMPC derived using approximation can stabilise the original general nonlinear system if certain condition is satisfied. Since no online optimisation is required, this provides an easy to implement suboptimal NMPC for general nonlinear systems, in particular, for nonlinear systems with fast dynamics. 


\section{Appendix}

\section{A 1: Proof of Theorem 1}

It follows from (17) and (19 that

$$
\begin{gathered}
\boldsymbol{Y}(t)-\boldsymbol{\omega}(t)=\left[\begin{array}{c}
\boldsymbol{M}_{\mu}(t) \\
\boldsymbol{H}(\boldsymbol{u}(t))
\end{array}\right] . \\
\boldsymbol{M}_{\mu}=\left[\begin{array}{c}
h(\boldsymbol{x})-\omega \\
\mathcal{D}_{\boldsymbol{f}_{x}}^{1} h(\boldsymbol{x})-\dot{\omega} \\
\vdots \\
\mathcal{D}_{\boldsymbol{f}_{x}}^{\mu} h(\boldsymbol{x})-\omega^{[\mu]}
\end{array}\right]
\end{gathered}
$$

and

$$
\boldsymbol{H}(\boldsymbol{u})=\left[\begin{array}{c}
\mathcal{D}_{\boldsymbol{f}_{x}}^{\mu+1} h(\boldsymbol{x})+\mathcal{D}_{u} \mathcal{D}_{\boldsymbol{f}_{x}} h(\boldsymbol{x}) \dot{u}-\omega^{[\mu+1]} \\
\vdots \\
\mathcal{D}_{\boldsymbol{f}_{x}}^{\mu+1} h(\boldsymbol{x})+\mathcal{D}_{u} \mathcal{D}_{\boldsymbol{f}_{x}} h(\boldsymbol{x}) u^{[r-\mu]}+z_{r-\mu-1}(\boldsymbol{x}, u, \boldsymbol{u})-\omega^{[r]}
\end{array}\right]
$$

Differentiating $\boldsymbol{H}(\boldsymbol{u})$ in (53) with respect to $\boldsymbol{u}$ gives

$$
\begin{aligned}
& \frac{\partial \boldsymbol{H}(\boldsymbol{u})}{\partial \boldsymbol{u}} \\
= & {\left[\begin{array}{ccccc}
\mathcal{D}_{u} \mathcal{D}_{\boldsymbol{f}_{x}}^{\mu-1} h(\boldsymbol{x}) & 0 & 0 & \ldots & 0 \\
\times & \mathcal{D}_{u} \mathcal{D}_{\boldsymbol{f}_{x}}^{\mu-1} h(\boldsymbol{x}) & 0 & \cdots & 0 \\
\vdots & \vdots & \vdots & \vdots & \vdots \\
\times & \times & \cdots & \times & \mathcal{D}_{u} \mathcal{D}_{\boldsymbol{f}_{x}}^{\mu-1} h(\boldsymbol{x})
\end{array}\right] }
\end{aligned}
$$

where ' $\times$ ' denotes the nonzero element in the matrix $\frac{\partial \boldsymbol{H}(\boldsymbol{u})}{\partial \boldsymbol{u}}$.

The necessary condition for the optimal control $\boldsymbol{u}$ is given by

$$
\frac{\partial J}{\partial \boldsymbol{u}}=0
$$

Partition the matrix $\mathcal{T}$ in (22) into the submatrices

$$
\mathcal{T}=\left[\begin{array}{cc}
\mathcal{T}_{\mu \mu} & \mathcal{T}_{\mu r} \\
\mathcal{T}_{\mu r}^{T} & \mathcal{T}_{r r}
\end{array}\right]
$$

where

$$
\mathcal{T}_{\mu \mu} \in \mathbb{R}^{\mu \times \mu}, \mathcal{T}_{\mu r} \in \mathbb{R}^{\mu \times(r+1)}, \mathcal{T}_{r r} \in \mathbb{R}^{(r+1) \times(r+1)}
$$


Differentiating the performance index $J$ in (21) with respect to control vector $\boldsymbol{u}$ and using the notations in (17), (52) and (54) yields

$$
\begin{aligned}
\frac{\partial J}{\partial \boldsymbol{u}} & =\left(\frac{\partial(\boldsymbol{Y}-\boldsymbol{\omega})}{\partial \boldsymbol{u}}\right)^{T} \mathcal{T}(\boldsymbol{Y}-\boldsymbol{\omega}) \\
& =\left[\begin{array}{c}
0 \\
\frac{\partial \boldsymbol{H}(\boldsymbol{u})}{\partial \boldsymbol{u}}
\end{array}\right]^{T} \mathcal{T}(\boldsymbol{Y}-\boldsymbol{\omega}) \\
& =\left[\begin{array}{ll}
0 & \left(\frac{\partial \boldsymbol{H}(\boldsymbol{u})}{\partial \boldsymbol{u}}\right)^{T}
\end{array}\right]\left[\begin{array}{cc}
\mathcal{T}_{\mu \mu} & \mathcal{T}_{\mu r} \\
\mathcal{T}_{\mu r}^{T} & \mathcal{T}_{r r}
\end{array}\right]\left[\begin{array}{c}
\boldsymbol{M}_{\mu} \\
\boldsymbol{H}(\boldsymbol{u})
\end{array}\right]
\end{aligned}
$$

It can be shown that the necessary condition (55) can be written as

$$
\left(\left.\frac{\partial \boldsymbol{H}(\boldsymbol{u})}{\partial \boldsymbol{u}}\right|_{\boldsymbol{u}=\boldsymbol{u}^{*}}\right)^{T} \mathcal{T}_{\mu r}^{T} \boldsymbol{M}_{\mu}+\left(\left.\frac{\partial \boldsymbol{H}(\boldsymbol{u})}{\partial \boldsymbol{u}}\right|_{\boldsymbol{u}=\boldsymbol{u}^{*}}\right)^{T} \mathcal{T}_{r r} \boldsymbol{H}\left(\boldsymbol{u}^{*}\right)=0
$$

where $\boldsymbol{u}^{*}$ denotes the solution satisfying the necessary optimality condition. Since $\mathcal{D}_{u} \mathcal{D}_{\boldsymbol{f}_{x}}^{\mu-1} h(\boldsymbol{x})$ is invertible according to Assumption A3, then $\frac{\partial \boldsymbol{H}(\boldsymbol{u})}{\partial \boldsymbol{u}}$ in (54) is also invertible. Note that the matrix $\mathcal{T}_{r r}$ is positive definite. Eq. (58) implies

$$
\boldsymbol{H}\left(\boldsymbol{u}^{*}\right)=-\mathcal{T}_{r r}^{-1} \mathcal{T}_{\mu r}^{T} \boldsymbol{M}_{\mu}
$$

Following (53), the first equations in (59) can be written as

$$
\mathcal{D}_{u} \mathcal{D}_{\boldsymbol{f}_{x}}^{\mu} h(\boldsymbol{x}) \dot{u}(t)^{*}+\boldsymbol{K} \boldsymbol{M}_{\mu}+\mathcal{D}_{\boldsymbol{f}_{x}}^{\mu+1} h(\boldsymbol{x})-\omega(t)^{[\mu+1]}=0
$$

where $\boldsymbol{K}$ denotes the first row of the matrix $\mathcal{T}_{r r}^{-1} \mathcal{T}_{\mu r}^{T}$. The optimal $\dot{u}(t)^{*}$ can be uniquely determined by

$$
\dot{u}(t)^{*}=-\left(\mathcal{D}_{u} \mathcal{D}_{\boldsymbol{f}_{x}}^{\mu} h(\boldsymbol{x})\right)^{-1}\left(\boldsymbol{K} \boldsymbol{M}_{\mu}(t)+\mathcal{D}_{\boldsymbol{f}_{x}}^{\mu+1} h(\boldsymbol{x})-\omega^{[\mu+1]}(t)\right)
$$

since this is the only solution to Eq. (59) and thus optimal condition (55).

In the moving time frame $t+\tau$ located at time $\mathrm{t}$, differentiating $u(t+\tau)$ in $(23)$ with respect to $\tau$ gives

$$
\frac{d u(t+\tau)}{d \tau}=\dot{u}(t)^{*}+\tau \ddot{u}(t)^{*} \ldots+\frac{\tau^{[r-\mu-1]}}{r-\mu-1 !} u^{[r-\mu]}(t)^{*}, \quad 0 \leq \tau \leq T_{2}
$$

In the implementation of NMPC,

$$
\dot{u}(t)=\frac{d u(t+\tau)}{d \tau} \quad \text { for } \tau=0
$$

The control law (27) is obtained by combining $(62), 63)$ and (61). 


\section{A2: Optimal control vector $\boldsymbol{u}(t)$}

Although in the implementation of the NMPC, only the optimal $\dot{u}$ is required, all variables to be optimised in $\boldsymbol{u}(t)$ can be uniquely determined by Eq. (59). Following (53), (12) and (13), the second equations in (59) can be written as

$$
\mathcal{D}_{u} \mathcal{D}_{\boldsymbol{f}_{x}}^{\mu} h(\boldsymbol{x}) \ddot{u}(t)^{*}+z_{1}\left(\boldsymbol{x}, u(t), \dot{u}(t)^{*}\right)+\mathcal{D}_{\boldsymbol{f}_{x}}^{\mu+2} h(\boldsymbol{x})-\omega^{[\mu+2]}(t)=-\boldsymbol{K}_{2} \boldsymbol{M}_{\mu}
$$

Hence the optimal $\ddot{u}(t)^{*}$ is determined by

$$
\ddot{u}(t)^{*}=-\left(\mathcal{D}_{u} \mathcal{D}_{\boldsymbol{f}_{x}}^{\mu} h(\boldsymbol{x})\right)^{-1}\left(\boldsymbol{K}_{2} \boldsymbol{M}_{\mu}+z_{1}\left(\boldsymbol{x}, u(t), \dot{u}(t)^{*}\right)+\mathcal{D}_{\boldsymbol{f}_{x}}^{\mu+2} h(\boldsymbol{x})-\omega^{[\mu+2]}(t)\right)
$$

where $\boldsymbol{K}_{2}$ denotes the second row in the matrix $\mathcal{T}_{r r}^{-1} \mathcal{T}_{\mu r}^{T}$. After the optimal control $\dot{u}(t)^{*}$ is obtained from $(61), \ddot{u}(t)^{*}$ can be calculated by substituting (61) into (65). Recursively, optimal $u^{[3]}(t)^{*}, \ldots, u^{[r-\mu]}(t)^{*}$ also can be uniquely determined from the other equations in (59).

\section{References}

[1] D.J. Leith and W.E. Leithhead. Gain-scheduled and nonlinear systems: dynamic analysis by velocity-based linearisation families. International Journal of Control, 70(2):289-317, 1998.

[2] G. Prasad, E. Swidenbank, and B.W. Hogg. A local model networks based multivariable long-range predictive control strategy for termal power plants. Automatica, 34(10):1185-1204, 1998.

[3] K.J. Hunt and T.A. Johansen. Design and analysis of gain-scheduled control using local controller networks. International Journal of Control, 66(5):619-651, 1997.

[4] R. Murray-Smith and T.A. Johansen, editors. Miltiple modelling approach to modelling and Control. Taylor and Francies, 1997.

[5] D.J. Leith and W.E. Leithhead. Gain-scheduled controller design: an analytic framework directly incoraporating non-equilibrium plant dynamics. International Journal of Control, 70:289-317, 1998. 
[6] I. Siller-Alcala. Generalised Predictive Control of Nonlinear Systems. Ph.D. Thesis, Glasgow University, Faculty of Engineering, 1998. Supervised by P. J. Gawthrop.

[7] P. J. Gawthrop, H. Demircioglu, and I. Siller-Alcala. Multivariable continuoustime generalised predictive control: A state-space approach to linear and nonlinear systems. IEE Proceedings Part-D: Control Theory and Applications, 145(3):241250, May 1998.

[8] H. Chen and F. Allgöwer. A quasi-infinite horizon nonlinear model predictive control scheme with guaranteed stability. Automatica, 34(10):1205-1217, 1998.

[9] S. L. de Oliveira Kothare and M. Morari. Contractive model predictive control for constrainted nonlinear systems. IEEE Transactions on Automatic Control, 45:1053-1071, 2000.

[10] F. Allgöwer and A. Zheng, editors. Nonlinear Model Predictive Control. Progress in Systems and Control Theory. Birkhäuser Verlag, 1998.

[11] P.J. Gawthrop and T. Arsan. Exact linearisation is a special case of non-linear GPC (abstract only). In F Allgöwer and A. Zheng, editors, Preprints of Int. Symposium on Nonlinear Model Predictive Control: Assessment and Future Directions, Ascona, Switzerland, page 37. Berlin: Springer, June 1998.

[12] M. Soroush and H. M. Soroush. Input-output linearising nonlinear model predictive control. Int. J. Control, 68(6):1449-1473, 1997.

[13] P. Lu. Optimal predictive control for continuous nonlinear systems. International Journal of Control, 62(3):633-649, 1995.

[14] W.-H. Chen, D.J. Ballance, and P. J. Gawthrop. Optimal control of nonlinear systems: A predictive control approach. Automatica, 39(4):633-641, 2003.

[15] W.-H. Chen. Analytic predictive controllers for nonlinear systems with ill-defined relative degree. IEE Proceedings Part D: Control Theory and Applications, 148(1):9-16, 2001.

[16] A. Isidori. Nonlinear Control Systems: An Introduction. 3rd Ed. Springer-Verlag, New York, 1995. 